\title{
THE CHANDRA PLANETARY NEBULA SURVEY (CHANPLANS). II. X-RAY EMISSION FROM COMPACT PLANETARY NEBULAE
}

\author{
M. Freeman ${ }^{1}$, R. Montez Jr. ${ }^{2}$, J. H. Kastner ${ }^{1}$, B. Balick ${ }^{3}$, D. J. Frew ${ }^{4}$, D. Jones ${ }^{5,6}$, B. Miszalski ${ }^{7,8}$, \\ R. Sahai ${ }^{9}$, E. Blackman ${ }^{10}$, Y.-H. Chu ${ }^{11}$, O. De Marco ${ }^{4}$, A. Frank ${ }^{10}$, M. A. Guerrero ${ }^{12}$, J. A. Lopez ${ }^{13}$, A. Zijlstra ${ }^{14}$, \\ V. Bujarrabal ${ }^{15}$, R. L. M. Corradi ${ }^{16,17}$, J. Nordhaus ${ }^{17}$, Q. A. PArker ${ }^{4,18}$, C. SAndiN ${ }^{19}$, D. SchönBerner ${ }^{19}$, \\ N. Soker ${ }^{20}$, J. L. Sokoloski ${ }^{21}$, M. StefFen ${ }^{19}$, J. A. Toalá ${ }^{12}$, T. Ueta ${ }^{22}$, And E. Villaver ${ }^{23}$ \\ ${ }^{1}$ Center for Imaging Science and Laboratory for Multiwavelength Astrophysics, Rochester Institute of Technology, \\ 54 Lomb Memorial Drive, Rochester, NY 14623, USA \\ ${ }^{2}$ Department of Physics \& Astronomy, Vanderbilt University, Nashville, TN, USA \\ ${ }^{3}$ Department of Astronomy, University of Washington, Seattle, WA, USA \\ ${ }^{4}$ Department of Physics \& Astronomy and Macquarie Research Centre for Astronomy, Astrophysics \& Astrophotonics, \\ Macquarie University, Sydney, NSW 2109, Australia \\ ${ }^{5}$ Departamento de Física, Universidad de Atacama, Copayapu 485, Copiapó, Chile \\ ${ }^{6}$ European Southern Observatory, Alonso de Córdova 3107, Casilla 19001, Santiago, Chile \\ ${ }^{7}$ South African Astronomical Observatory, P.O. Box 9, Observatory, 7935, South Africa \\ ${ }^{8}$ Southern African Large Telescope Foundation, P.O. Box 9, Observatory, 7935, South Africa \\ 9 Jet Propulsion Laboratory, MS 183-900, California Institute of Technology, Pasadena, CA 91109, USA \\ ${ }^{10}$ Department of Physics \& Astronomy, University of Rochester, Rochester, NY, USA \\ ${ }^{11}$ Department of Astronomy, University of Illinois at Urbana-Champaign, Urbana, IL, USA \\ ${ }^{12}$ Instituto de Astrofísica de Andalucía, IAA-CSIC, Glorieta de la Astronomía s/n, Granada, E-18008, Spain \\ ${ }^{13}$ Instituto de Astronomía, Universidad Nacional Autonoma de Mexico, Campus Ensenada, Apdo. Postal 22860, Ensenada, B. C., Mexico \\ ${ }^{14}$ School of Physics and Astronomy, University of Manchester, Manchester M13 9PL, UK \\ ${ }^{15}$ Instituto de Astrofísica de Canarias, E-38200 La Laguna, Tenerife, Spain \\ ${ }^{16}$ Departamento de Astrofísica, Universidad de La Laguna, E-38206 La Laguna, Tenerife, Spain \\ ${ }^{17}$ NSF Astronomy and Astrophysics Fellow, Center for Computational Relativity and Gravitation, \\ Rochester Institute of Technology, Rochester, NY 14623, USA \\ 18 Australian Astronomical Observatory, P.O. Box 296, Epping, NSW 2121, Australia \\ ${ }^{19}$ Leibniz Institute for Astrophysics Potsdam (AIP), An der Sternwarte 16, D-14482 Potsdam, Germany \\ ${ }^{20}$ Observatorio Astronomico Nacional, Apartado 112, E-28803, Alcala de Henares, Spain \\ ${ }^{21}$ Columbia Astrophysics Laboratory, Columbia University, New York, NY 10027, USA \\ 22 Department of Physics \& Astronomy, University of Denver, Denver, CO 80208, USA \\ ${ }^{23}$ Departamento de Física Teórica, Universidad Autónoma de Madrid, Cantoblanco, E-28049 Madrid, Spain \\ Received 2014 May 2; accepted 2014 August 8; published 2014 September 26
}

\begin{abstract}
We present results from the most recent set of observations obtained as part of the Chandra X-ray observatory Planetary Nebula Survey (CHAnPlaNS), the first comprehensive X-ray survey of planetary nebulae (PNe) in the solar neighborhood (i.e., within $\sim 1.5 \mathrm{kpc}$ of the Sun). The survey is designed to place constraints on the frequency of appearance and range of X-ray spectral characteristics of X-ray-emitting PN central stars and the evolutionary timescales of wind-shock-heated bubbles within PNe. CHANPLANS began with a combined Cycle 12 and archive Chandra survey of 35 PNe. CHAnPlaNS continued via a Chandra Cycle 14 Large Program which targeted all (24) remaining known compact $\left(R_{\text {neb }} \lesssim 0.4 \mathrm{pc}\right)$, young PNe that lie within $\sim 1.5 \mathrm{kpc}$. Results from these Cycle 14 observations include first-time X-ray detections of hot bubbles within NGC 1501, 3918, 6153, and 6369, and point sources in HbDs 1, NGC 6337, and Sp 1. The addition of the Cycle 14 results brings the overall CHANPLANS diffuse X-ray detection rate to $\sim 27 \%$ and the point source detection rate to $\sim 36 \%$. It has become clearer that diffuse X-ray emission is associated with young $\left(\lesssim 5 \times 10^{3} \mathrm{yr}\right)$, and likewise compact $\left(R_{\text {neb }} \lesssim 0.15 \mathrm{pc}\right)$, PNe with closed structures and high central electron densities $\left(n_{e} \gtrsim 1000 \mathrm{~cm}^{-3}\right)$, and is rarely associated with PNe that show $\mathrm{H}_{2}$ emission and/or pronounced butterfly structures. Hb 5 is one such exception of a PN with a butterfly structure that hosts diffuse X-ray emission. Additionally, two of the five new diffuse X-ray detections (NGC 1501 and NGC 6369) host [WR]-type central stars, supporting the hypothesis that PNe with central stars of [WR]-type are likely to display diffuse X-ray emission.
\end{abstract}

Key words: binaries: general - planetary nebulae: individual (NGC 1501, NGC 3918, NGC 6153, NGC 6369, HbDs 1, NGC 6337, Sp 1) - stars: AGB and post-AGB

Online-only material: color figures

\section{INTRODUCTION}

Planetary nebulae $(\mathrm{PNe})$, which represent the near-ends of the lives of intermediate-mass $\left(\sim 1-8 M_{\odot}\right)$ stars, afford insight into the late stages of stellar evolution. As an asymptotic giant branch (AGB) star sheds its tenuous envelope, it unveils a hot ( $T$ up to $\gtrsim 10^{5} \mathrm{~K}$ ) core that is capable of ionizing the surrounding material and will eventually evolve into a white dwarf (WD). $\mathrm{PNe}$ are well known for their wide variety of optical emission line morphologies; this morphological diversity reflects the effects of energetic, nebula-shaping wind collisions. Recently, $\mathrm{X}$-ray observations of $\mathrm{PNe}$ have shed light on the processes within nebular interiors, as well as on the properties of their central stars, that directly relate to PN shaping (for a review, see Kastner et al. 2012, hereafter Paper I).

Models describing the structure of PNe (Kwok et al. 1978; Schmidt-Voigt \& Koppen 1987; Marten \& Schönberner 1991; Villaver et al. 2002; Perinotto et al. 2004; Toalá \& Arthur 2014) 
posit that fast ( $v_{\mathrm{w}} \simeq 500-1500 \mathrm{~km} \mathrm{~s}^{-1}$ ) winds emanating from the pre-WD will collide with the previously ejected AGB envelope, sweeping up the AGB ejecta into a thin shell. These wind collisions result in shocks that heat the fast wind gas to temperatures $>10^{6} \mathrm{~K}$, creating a "hot bubble" capable of producing soft X-rays $(0.3-1 \mathrm{keV})$. A binary companion to the central star of the PN (CSPN) is capable of further influencing the PN shape via the formation of an accretion disk (Soker \& Rappaport 2000), the transfer of angular momentum, the generation of a strong magnetic dynamo at the CSPN (Nordhaus et al. 2007), or, in the case of a close binary, the formation of jets (Soker \& Livio 1994; Miszalski et al. 2011; Corradi et al. 2011; Boffin et al. 2012; Tocknell et al. 2014). While some CSPNe with hot photospheres $(\gtrsim 100 \mathrm{kK})$ are capable of emitting soft $\mathrm{X}$-rays, many CSPNe exhibit hard X-rays $(>1 \mathrm{keV})$ that cannot be attributed to photospheric emission alone (Montez \& Kastner 2013; Montez et al. 2014). Such hard X-rays might be expected, however, when a hot, compact companion accretes material, as suggested for some WDs with hard X-ray emission (Bilíková et al. 2010), or from the corona of a "rejuvenated" late-type companion of a post-common envelope binary CSPN (Montez et al. 2010). There is also the possibility that a "hard excess" could result from material infall from a debris disk around the CSPN, similar to the debris disk discovered around the central star of the Helix Nebula and possibly linked to its hard X-ray emission (Su et al. 2007). Alternatively, the hard X-ray emission might emerge from shocks within the stellar wind, via a process similar to that observed in O and B stars (Cassinelli et al. 1994).

The Chandra Planetary Nebulae Survey (CHanPlaNS) is designed to place constraints on the frequency of the appearance and range of X-ray spectral characteristics of X-ray-emitting $\mathrm{PN}$ central stars and the evolutionary timescales of wind-shockheated bubbles within PNe. The initial CHANPLANS program consisted of observations of 21 high-excitation PNe selected from among the $\sim 120$ known PNe within $\sim 1.5 \mathrm{kpc}$ of Earth, along with another 14 PNe previously observed by Chandra that fall within this volume (Paper I). The initial results included more than a dozen new detections of X-ray point sources and placed new constraints on the physical conditions, timescales, and frequency of hot bubbles. The survey recently continued with a $670 \mathrm{ks}$ Chandra Cycle 14 Large Program of an additional 24 compact $\left(R_{\text {neb }} \lesssim 0.4 \mathrm{pc}\right)$ PNe selected from among the volume-limited sample. In this paper, we describe the results of the CHANPLANS survey observations of these compact PNe in the context of the overall volume-limited sample of PNe. In two companion papers (Montez et al. 2014, 2014, in preparation), we detail the characteristics of point-like and diffuse X-ray emission sources within $\mathrm{PNe}$, and further examine the origins and implications of these PN X-ray sources.

\section{OBSERVATIONS AND DATA REDUCTION}

\subsection{Sample: Compact $\left(R_{\mathrm{neb}} \lesssim 0.4 p c\right)$ Planetary Nebulae within $\sim 1.5 \mathrm{kpc}$}

The PNe that constitute the sample observed with Chandra during Cycle 14, listed in Table 1, were primarily selected so as to further probe the onset, evolutionary timescale, and properties of diffuse X-ray sources within PNe. Hence, we selected objects on the basis of their relatively small physical dimensions, as ascertained via optical imaging. More specifically, we chose the 24 Table 1 objects from the compilation of solarneighborhood PNe in Frew (2008) such that the resulting merged Cycle 12+14+archival sample would constitute a representative volume-limited ( $D \lesssim 1.5 \mathrm{kpc}$ ) sample of PNe with inner bubble

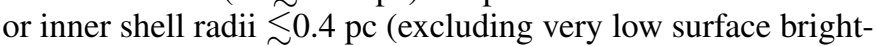
ness PNe, which typically have faint central stars). We estimate that our completeness in surveying compact $\left(R_{\text {neb }} \lesssim 0.4 \mathrm{pc}\right)$ nebulae within $\sim 1.5 \mathrm{kpc}$ is $90 \%$, based on the solar neighborhood census of PNe recently compiled by Frew et al. (2014b). The chosen nebular radius roughly corresponds to a dynamical PN lifetime of $\lesssim 10^{4} \mathrm{yr}$-approximately twice the apparent hot bubble dissipation timescale inferred from Cycle 12 plus archival Chandra data shown in Paper I-assuming typical PN expansion velocities of $\sim 40 \mathrm{~km} \mathrm{~s}^{-1}$ (Jacob et al. 2013). Basic PN and CSPN data for the sample objects can be found in Table 1 .

\subsection{Observations}

All Cycle 14 PNe were observed with the back-illuminated (BI) CCD S3 of Chandra's Advanced CCD Imaging Spectrometer (ACIS). The use of CCDs as X-ray detectors provides determinations of incident photon energies as well as locations, which in turn allows for filtering of images by photon energy. Chandra/ACIS-S3 has an energy sensitivity of $\sim 0.3-8.0 \mathrm{keV}$, with a field of view of $\sim 8^{\prime} \times 8^{\prime}$ and a pixel size of $0^{\prime \prime} .492$. The BI CCD S3 has greater low-energy ( $<1 \mathrm{keV})$ sensitivity compared to the front-illuminated ACIS-I array, extending sensitivity down to $\sim 0.2 \mathrm{keV}$ for high soft photon fluxes, albeit with uncertain calibration (Montez \& Kastner 2013). Additionally, the use of S3 facilitates subpixel event repositioning (SER) in processing so as to better sample the core of the point spread function generated by Chandra's High-Resolution Mirror Assembly ( $\mathrm{Li}$ et al. 2004). Observation IDs, dates, and exposure times for the Cycle 14 ChanPlaNS observations are listed in Table 2.

\subsection{Data Reduction}

All data were reduced using $\mathrm{CIAO}^{24}$ (version 4.5). Data reduction made use of the CHANPLANS pipeline, which is detailed in Paper I. Briefly, this pipeline consists of processing the following steps: reprocessing of the primary and secondary data files and the application of SER using chandra_repro; detection of sources in the full field of view of each observation using wavdetect; calculation of statistics from a 3".5 radius region centered on the CSPN, to place constraints on possible X-ray point source emission; and generation of annotated two-panel images displaying a filtered Chandra X-ray image highlighting the predominately soft X-ray $(0.3-2.0 \mathrm{keV})$ nature of the nebulae and the best available optical (H $\alpha$ or $R$ band) image obtained from the Hubble Legacy Archive, ${ }^{25}$ the WisconsinIndiana-Yale-NOAO (WIYN) 0.9 m telescope (see Paper I), the SuperCOSMOS H-Alpha Survey (Parker et al. 2005; Frew et al. 2014a), or the Digitized Sky Survey $\left(\mathrm{DSS}^{26}\right)$, as indicated in the panels (Figure 1).

\section{RESULTS}

Results from the Cycle 14 Chandra observations listed in Table 2 are illustrated in Figures 1-3 and are summarized in Tables 1 and 3. The last column of Table 1 indicates whether the PN was detected and, if so, the type of X-ray emission detected (point-like, diffuse, or both). As in Paper I, our conservative estimate for our sensitivity limits for diffuse and hard (soft) point-like X-ray sources are $\sim 10^{30}$ and $\sim 10^{29}\left(\sim 10^{31}\right) \mathrm{erg} \mathrm{s}^{-1}$,

\footnotetext{
24 Chandra Interactive Analysis of Observations; http://cxc.harvard.edu/ciao/

25 http://archive.stsci.edu/hst

$26 \mathrm{http} / / /$ archive.stsci.edu/dss
} 
Table 1

Planetary Nebulae within $1.5 \mathrm{kpc}^{\mathrm{a}}$ Observed by Chandra

\begin{tabular}{|c|c|c|c|c|c|c|c|c|c|c|}
\hline Name & PN G & $\begin{array}{c}\text { Morph. }^{\text {b }} \\
\text { (F08/SMV11) }\end{array}$ & $\begin{array}{c}D \\
(\mathrm{kpc})\end{array}$ & $\begin{array}{l}R_{\text {neb }} \\
(\mathrm{pc})\end{array}$ & $\begin{array}{c}\text { Age } \\
\left(10^{3} \mathrm{yr}\right)\end{array}$ & $\begin{array}{c}T_{*} \\
(\mathrm{kK})\end{array}$ & Sp. Type & Comp. ${ }^{\mathrm{c}}$ & $\mathrm{H}_{2}{ }^{\mathrm{d}}$ & X-Rays ${ }^{\mathrm{e}}$ \\
\hline A65 & $017.3-21.9$ & Eafm:/Ls & 1.17 & 0.30 & $17.5^{\mathrm{f}}$ & $114^{\mathrm{g}}$ & $\mathrm{O}(\mathrm{H})$ & $(\mathrm{Y})$ & $\ldots$ & $\mathrm{N}$ \\
\hline HaWe 13 & $034.1-10.5$ & Efp?/Esip & 1.01 & 0.18 & $\ldots$ & 68 & $\operatorname{hgO}(\mathrm{H})$ & $\ldots$ & $\ldots$ & $\mathrm{N}$ \\
\hline $\mathrm{Hb} 5$ & $359.3-00.9$ & Bps/Bcbmph & 1.70 & 0.13 & $1.5^{\mathrm{h}}$ & 172 & $\ldots$ & $\ldots$ & $\mathrm{Y}$ & $\mathrm{D}$ \\
\hline HbDs 1 & $273.6+06.1$ & Er?/Is & 0.80 & 0.29 & $\ldots$ & $119^{i}$ & $\mathrm{O}(\mathrm{H})$ & $\ldots$ & $\ldots$ & $\mathrm{P}$ \\
\hline He 2-11 & $259.1+00.9$ & Ebps/Bcbsip & 1.14 & 0.24 & $0.7^{\mathrm{j}}$ & $108^{\mathrm{j}}$ & $\ldots$ & $\mathrm{K} 5 \mathrm{~V}^{\mathrm{j}}$ & $\ldots$ & $\mathrm{N}$ \\
\hline IC 1295 & $025.4-04.7$ & Efm:/Es(b/ti) & 1.23 & 0.30 & 11 & 98 & $\operatorname{hgO}(\mathrm{H})$ & $\ldots$ & $\ldots$ & $\mathrm{N}$ \\
\hline IC 2149 & $166.1+10.4$ & $\mathrm{E} / \mathrm{Bsh}$ & 1.52 & 0.04 & 2 & 42 & $\mathrm{Of}(\mathrm{H})$ & $\ldots$ & $\mathrm{N}$ & $\mathrm{N}$ \\
\hline IC 4637 & $345.4+00.1$ & Eam/Estp & 1.30 & 0.05 & 2 & 50 & $\mathrm{O}(\mathrm{H})$ & $(\mathrm{Y} ?)$ & $\ldots$ & $\mathrm{N}$ \\
\hline IC $5148 / 50$ & $002.7-52.4$ & $\mathrm{Rm} / \mathrm{Rsiph}$ & 0.85 & 0.27 & 5 & 110 & $\operatorname{hgO}(\mathrm{H})$ & $\ldots$ & $\ldots$ & $\mathrm{N}$ \\
\hline M 1-26 & $358.9-00.7$ & R/Mcbsih & 1.20 & 0.02 & 1 & 33 & $\mathrm{Of}(\mathrm{H})$ & $\ldots$ & $\ldots$ & $\mathrm{N}$ \\
\hline M 1-41 & $006.7-02.2$ & $\mathrm{Bs} /(\mathrm{I} / \mathrm{B})$ & 1.47 & 0.15 & $\ldots$ & 187 & $\ldots$ & $\ldots$ & $\mathrm{Y}$ & $\mathrm{N}$ \\
\hline NGC 1501 & $144.5+06.5$ & Es/Esph & 0.72 & 0.09 & 2 & 135 & [WC4] & $\ldots$ & $\ldots$ & $\mathrm{D}$ \\
\hline NGC 2899 & $277.1-03.8$ & Baps/Bosbp & 1.37 & 0.37 & 14 & $215^{\mathrm{k}}$ & $\ldots$ & F5V: & $\mathrm{Y}$ & $\mathrm{N}$ \\
\hline NGC 3918 & $294.6+04.7$ & Ems(h)/Lsbairh & 1.84 & 0.08 & 3 & 150 & $\mathrm{O}(\mathrm{H}) ?$ & $\ldots$ & $\ldots$ & $\mathrm{D}$ \\
\hline NGC 6026 & $341.6+13.7$ & $\mathrm{Ef} / \mathrm{Ish}$ & 1.31 & 0.16 & 6 & 35 & $\mathrm{O} 7(\mathrm{H})$ & WD? & $\ldots$ & $\mathrm{N}$ \\
\hline NGC 6072 & $342.1+10.8$ & $\mathrm{Ba} / \mathrm{Mcot}$ & 1.39 & 0.23 & 23 & 140 & $\ldots$ & $\ldots$ & $\mathrm{Y}$ & $\mathrm{N}$ \\
\hline NGC 6153 & $341.8+05.4$ & Es/Esah & 1.10 & 0.07 & 4 & 109 & $\mathrm{Of}(\mathrm{H}) ?$ & $\ldots$ & $\mathrm{N}$ & $\mathrm{D}$ \\
\hline NGC 6337 & $349.3-01.1$ & Epr/Rsarh & 0.86 & 0.10 & 12 & 105 & $\ldots$ & $(\mathrm{Y})$ & $\ldots$ & $\mathrm{P}$ \\
\hline NGC 6369 & $002.4+05.8$ & Ebpr(h:)/Mcst & 1.55 & 0.12 & 3 & 66 & [WO3] & $\ldots$ & $\mathrm{Y}$ & $\mathrm{D}$ \\
\hline NGC 6894 & $069.4-02.6$ & Emr/Eh & 1.31 & 0.17 & 4 & 100 & $\ldots$ & $\ldots$ & $\ldots$ & $\mathrm{N}$ \\
\hline NGC 7076 & $101.8+08.7$ & $\mathrm{Ea} / \mathrm{Esh}$ & 1.47 & 0.20 & 5 & 80 & $\ldots$ & $\ldots$ & $\ldots$ & $\mathrm{N}$ \\
\hline NGC 7354 & $107.8+02.3$ & Emp/Esaph & 1.60 & 0.09 & 3 & 96 & $\ldots$ & $\ldots$ & $\mathrm{N}$ & $\mathrm{N}$ \\
\hline Sh 2-71 & $035.9-01.1$ & Bs/Lsbp & 1.14 & 0.30 & 14 & 157 & $\ldots$ & $\ldots$ & $\mathrm{N}$ & $\mathrm{N}$ \\
\hline Sp 1 & $329.0+01.9$ & $\mathrm{Rr} / \mathrm{Rsh}$ & 1.13 & 0.20 & 6 & 72 & $\mathrm{O}(\mathrm{H})$ & $(\mathrm{Y})$ & $\ldots$ & $\mathrm{P}$ \\
\hline
\end{tabular}

Notes.

${ }^{a} \mathrm{PN}$ and central star data compiled from (Frew 2008, and references therein) unless otherwise noted.

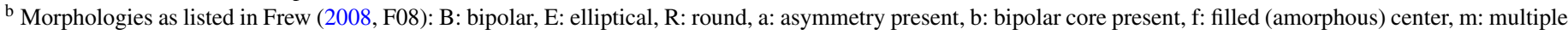

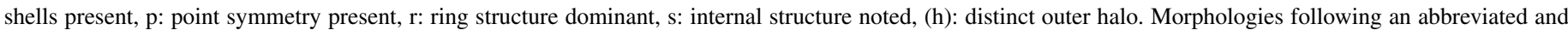

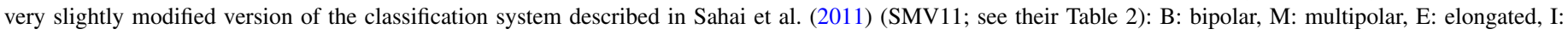

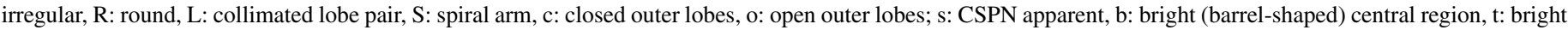
central toroidal structure; p: point symmetry, a: ansae, i: inner bubble, h: halo; r: radial rays; (/): alternate possibilities.

c " $(\mathrm{Y})$ " = known binaries with unknown companion types; "(Y?)" = possible binary; otherwise the type of the companion star is as listed (De Marco 2009).

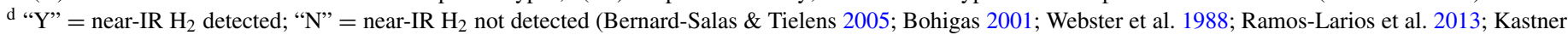
et al. 1996, and references therein).

e $\mathrm{X}$-ray results key: $\mathrm{P}=$ point source; $\mathrm{D}=$ diffuse source; $\mathrm{N}=$ not detected.

${ }^{\mathrm{f}}$ Huckvale et al. (2013).

g Hillwig et al. (2014).

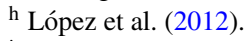

${ }^{\mathrm{i}}$ Herald \& Bianchi (2011).

j Jones et al. (2014a).

${ }^{\mathrm{k}}$ Drew et al. (2014).

respectively, at a distance of $1.5 \mathrm{kpc}$. We will revisit these sensitivity limits in Montez et al. (2014, 2014, in preparation).

Table 4 (an update of Table 4 from Paper I) lists X-ray detection statistics broken down by PN morphology, $\mathrm{H}_{2}$, detection/non-detection fraction, and known central star binarity for the entire ChanPlaNS sample. Figures 3 and 4 are updates of Figures 5 and 6, respectively, in Paper I. In Figure 3, various observed parameters for the sample $\mathrm{PNe}$-optical morphology, $\mathrm{H}_{2}$ detection/non-detection, $\mathrm{X}$-ray detection/nondetection, and X-ray source type-are illustrated in a plot of CSPN effective temperature versus nebular radius. In Figure 4, we plot median X-ray photon energy against nebular radius. In Figure 5, which has no counterpart in Paper I, we further illustrated PN X-ray detections and non-detections in the form of plots of PN distances and ratios of [O III] to $\mathrm{H} \beta$ fluxes against nebular densities for all (59) CHANPLANS sample PNe.

\subsection{Cycle 14 Detections of X-Rays from $P N e$}

We have detected five of the Table $1 \mathrm{PNe}$ in diffuse Xray emission: Hb 5, NGC 1501, NGC 3918, NGC 6153, and
NGC 6369 (Figure 2). The last four objects are detected for the first time; Hb 5 was detected previously by XMM-Newton (Montez et al. 2009, see below). These detections represent a $\sim 21 \%(5 / 24)$ detection rate of hot bubbles within the Cycle 14 sample. When these detections are combined with the previous ChanPlaNS observations, the CHANPlaNS detection rate of PNe hot bubbles now stands at $\sim 27 \%(16 / 59)$. Two of the newly detected objects, NGC 1501 and NGC 6369, host WolfRayet-type (WR) CSPNe, which brings the ChanPlaNS diffuse detection rate of such objects to $100 \%(5 / 5)$. The detection rate of diffuse X-ray emission from [WR]-type CSPNe remains high when including PNe beyond $\sim 1.5 \mathrm{kpc}$ (see Kastner et al. 2008). These diffuse X-ray detections follow the previously established trend that diffuse X-ray PNe generally display compact $\left(R_{\text {neb }} \lesssim\right.$ $0.15 \mathrm{pc}$ ), elliptical optical morphologies (Figure 3; Paper I). A notable exception is $\mathrm{Hb} 5$, which has a bipolar morphology.

The detections of X-ray point sources within three Cycle 14 PNe, HbDs1, NGC 6337, and Sp 1 (Table 1) yielded a $\sim 13 \%$ detection rate of CSPNe. These Cycle 14 detections bring the overall CHANPLANS detection rate of CSPNe to $236 \%(21 / 59)$. 

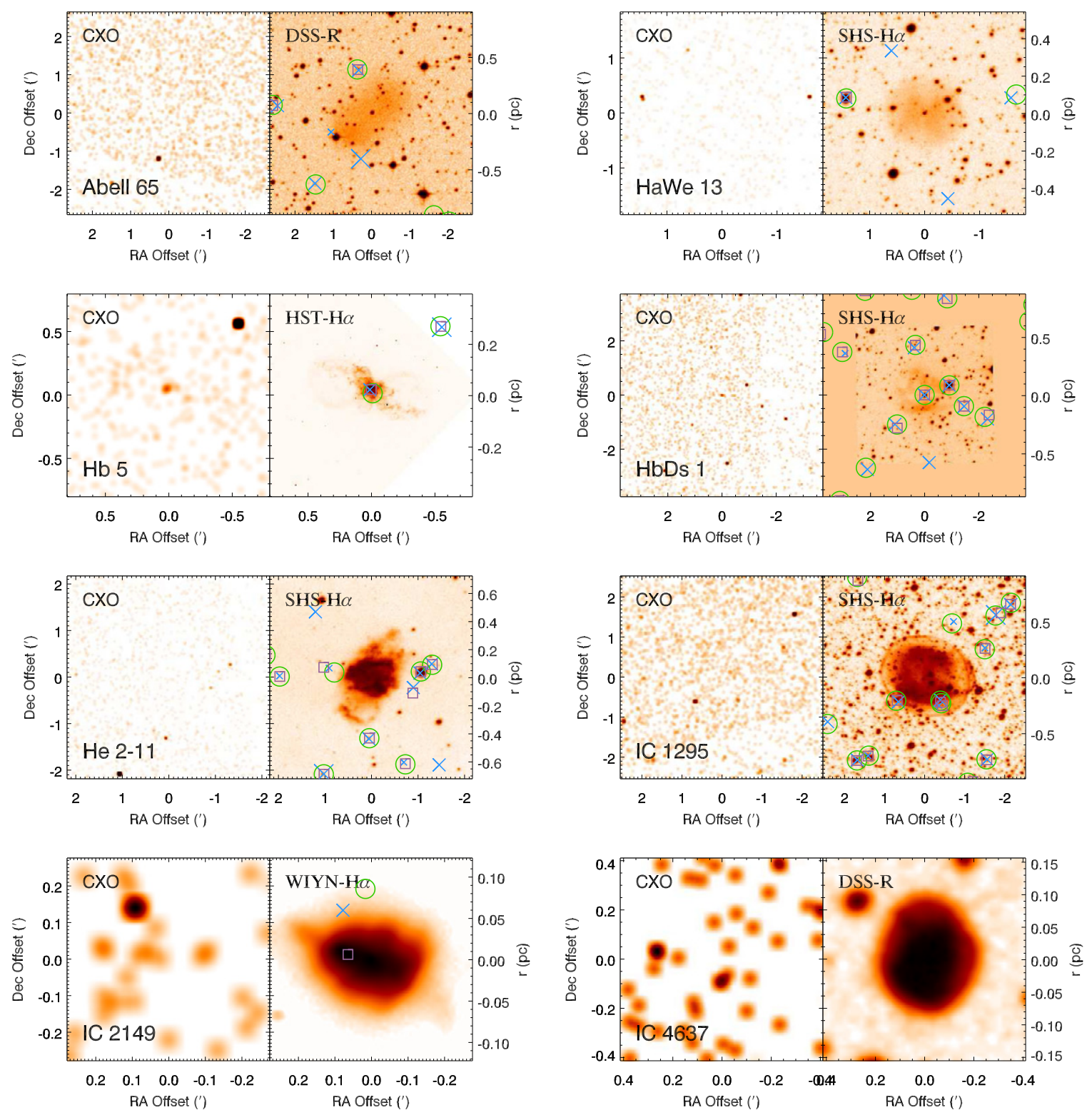

Figure 1. ChanPlaNS pipeline output for the Table 1 PNe. Two panels are presented for each PN. The left panel of each pair shows the Chandra/ACIS soft-band (0.3-2.0) keV image, smoothed with a Gaussian function with $3^{\prime \prime}$ FWHM (1" FWHM for M 1-26, or 6" FWHM for images larger than $5^{\prime}$ on a side), centered on the SIMBAD coordinates of the PN (which lies on back-illuminated CCD S3). The right panel shows an optical image (obtained from HST, WIYN, the SuperCOSMOS $\mathrm{H}$-alpha Survey, or the DSS, as indicated) overlaid with the positions of detected broad-band (0.3-8.0 keV) X-ray sources (crosses), USNO catalog stars (circles), and 2MASS Point Source Catalog IR sources (squares). The size of the cross is proportional to the number of X-ray photons detected.

(A color version of this figure is available in the online journal.)

Two of the three new CSPNe detections (within NGC 6337 and Sp 1) are known binaries, which brings the overall CHANPLANS detection rate of all CSPN that are known to be binaries (at the time this paper was written) to $60 \%$ (Table 4).

\subsection{PNe Displaying Diffuse X-Ray Emission}

The diffuse X-ray emitting PNe detected in the Cycle 14 sample are displayed in Figure 2 and are briefly described below. As is evident in Figures 3 and 4 and Table 4, these detections reinforce previous CHANPLANS results indicating that $\mathrm{PNe}$ displaying diffuse $\mathrm{X}$-rays are predominantly elliptical in morphology with $R_{\text {neb }} \lesssim 0.15 \mathrm{pc}$; furthermore, diffuse $\mathrm{X}$-ray $\mathrm{PNe}$ generally lack detections of near-IR $\mathrm{H}_{2}$ emission. In a forthcoming paper (R. Montez et al. 2014, in preparation), we present a global analysis of the CHANPLANS diffuse X-ray sources. Here, we describe the properties of the diffuse X-ray PNe detected during Cycle 14 observations.

\subsubsection{Classical Hot Bubbles}

NGC 3918 appears to host multiple shells, and is similar in optical and X-ray morphology to multiple-shell, diffuse X-ray PNe such as NGC 6543 (Paper I). Three-dimensional models of the nebula vary in their interpretations of its morphology. Clegg et al. (1987) modeled NGC 3918 as a biconical nebula within an optically thin sphere, while a spindle-like structure was favored by both Corradi et al. (1999) and Ercolano et al. (2003). Like NGC 1501, the shell of the nebula shows enhanced [O III]/H $\alpha$ emission resulting from the inner shell expanding into the outer shell (Guerrero et al. 2013), while the inner shell confines the diffuse X-ray emission. 

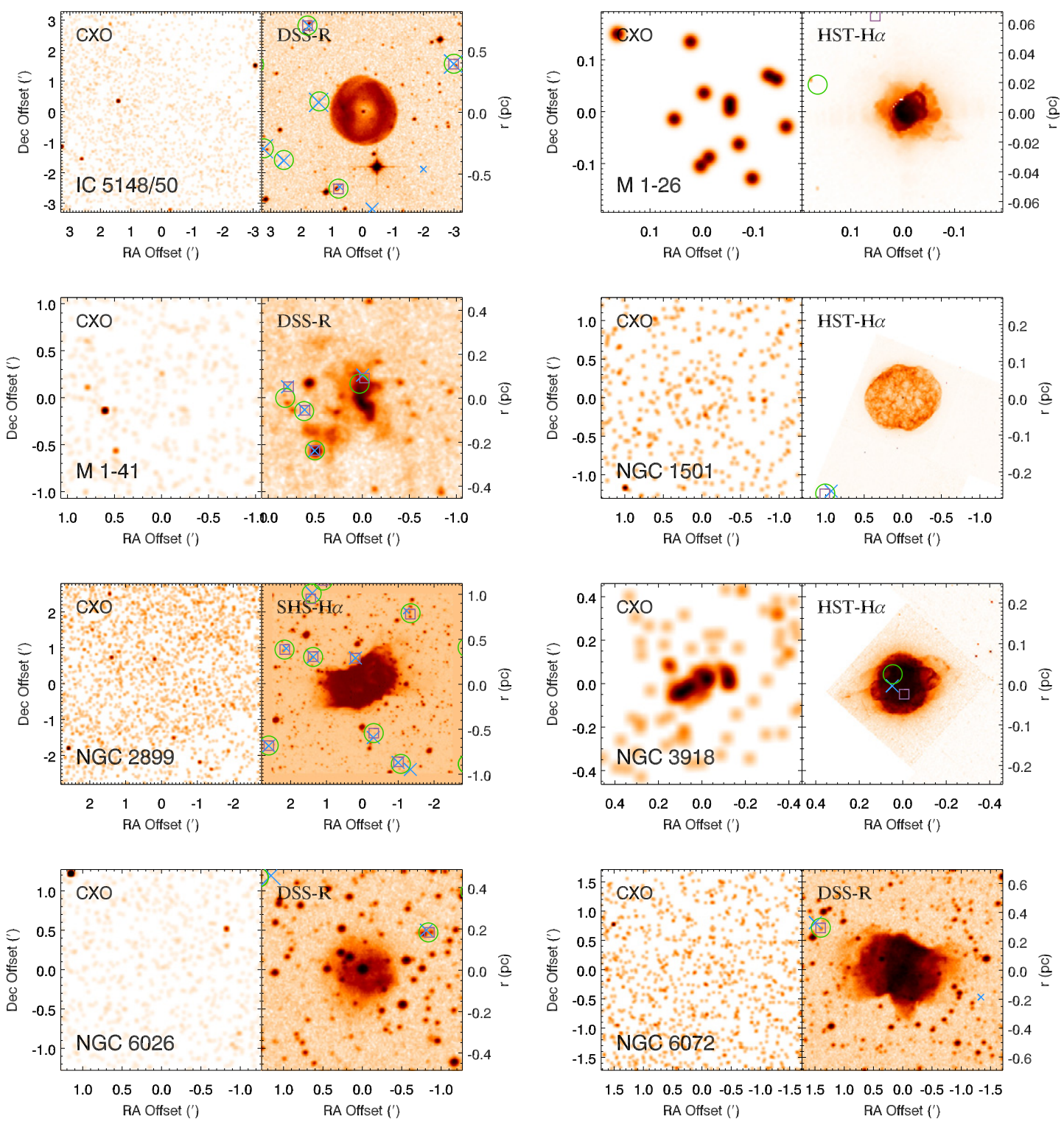

Figure 1. (Continued)

NGC 6153 is also an elliptical nebula that hosts multiple shells, and is thought to be the product of a high-mass $\left(4.5 M_{\odot}\right)$ progenitor (Pottasch \& Bernard-Salas 2010). Often compared to NGC 7009, NGC 6153 is indeed morphologically similar, both in the optical and X-ray; however, NGC 6153 lacks the ansae found in NGC 7009. The diffuse X-ray emission is confined to the inner shell, as is the case with NGC 3918 and similar multiple-shell, diffuse X-ray PNe (Paper I).

\subsubsection{PNe with [WR]-type CSPNe}

Both NGC 1501 and NGC 6369 harbor [WR]-type central stars and diffuse X-ray emission. NGC 1501 is an elliptical nebula with a [WR]-type central star within a complex, filamentary internal structure. The central star has been reclassified many times between [WO] and [WC] type. Based on emission lines detected in the optical spectrum, the CSPN appears to be [WO4] (Crowther et al. 1998; Ercolano et al. 2004); however, the star also displays an infrared excess from $J$ through all Spitzer IRAC bands, which is indicative of circumstellar dust, as is common for [WC]-type stars (Bilíková et al. 2012). NGC 1501 shows enhanced $[\mathrm{O}$ III $] / \mathrm{H} \alpha$ emission along the surface of the nebular shell, which is evidence for shocks along the skin (Guerrero et al. 2013). Similarly, the X-ray emission appears to trace shocks inside the primary nebular shell.

In contrast to the elliptical shell and filamentary internal structure of NGC 1501, NGC 6369 appears to have a spherical main nebular shell described as a barrel, with bipolar outflows (Ramos-Larios et al. 2012). This structure is similar to that of NGC 40 (Montez et al. 2005). Where [O III] and $\mathrm{H} \alpha$ are dim in NGC 6369, the nebula is bright in [N II], displaying a ring of emission along the inner shell. The bright optical shell is filled with $\mathrm{H}_{2}$ and surrounded by a photodissociation region (RamosLarios et al. 2013), and the shell encloses the diffuse X-ray emission around the [WO]-type central star (Peña et al. 2013). The correspondence of the X-ray emission and the nebular shell, and lack of emission from the blowouts, suggests that emission is generated by the strong winds of the CSPN colliding with the progenitor red giant wind, as is the case for NGC 40 (Montez et al. 2005). 

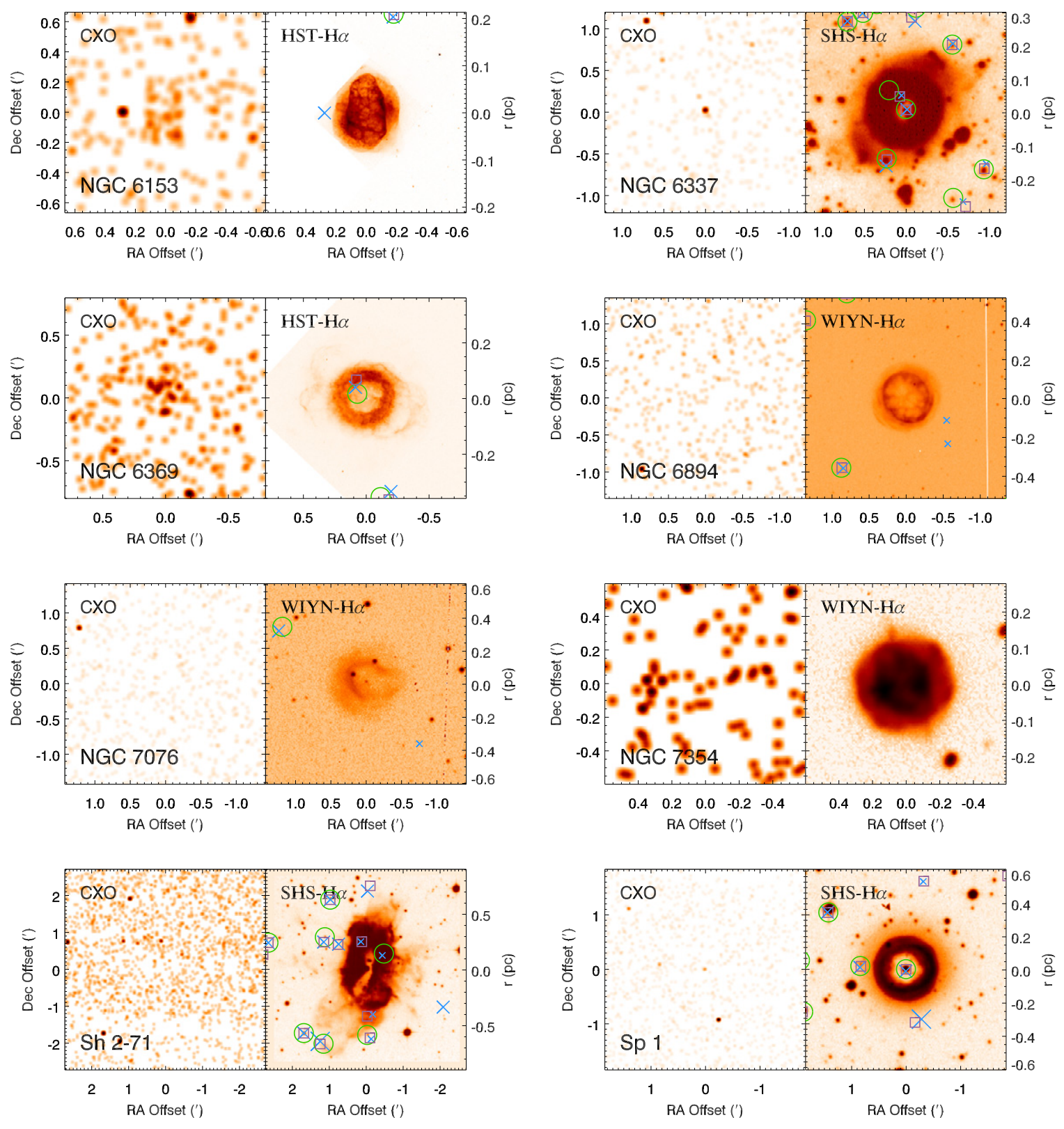

Figure 1. (Continued)

\subsubsection{The Bipolar Planetary Nebula, Hb 5}

$\mathrm{Hb} 5$ is a bipolar nebula with large lobes $\left(\sim 27^{\prime \prime}\right)$ extending east and west and a tight waist with a bright core that contains secondary expanding lobes. The smaller lobes $\left(\lesssim 12^{\prime \prime}\right)$ may result from an interaction between a fast, low-density wind and an inhomogeneous, high-density circumstellar shell (López et al. 2012). Diffuse X-ray emission was previously detected serendipitously by XMM-Newton (Montez et al. 2009). The emission in the $X M M$ images appeared coincident with the peak optical emission around the core and possibly along the $\mathrm{H} \alpha$ emission in the lobes, although $X M M$ 's resolution was insufficient to determine if the X-ray emission is extended.

The Cycle 14 Chandra observations of $\mathrm{Hb} 5$ also yielded the detection of a bright, compact X-ray core, coincident with the peak optical emission. There is no apparent X-ray point source from the CSPNe contributing to the core emission. Additionally, $\mathrm{X}$-ray emission was tentatively detected along the tip of the eastern lobe, which appears blueshifted, while no apparent emission is seen from the western, redshifted lobe, which may be attributed to extinction by intervening nebular material (López et al. 2012).

\subsection{PNe Displaying Point-like X-Ray Emission at Central Stars}

The three Cycle $14 \mathrm{X}$-ray point source detections (HbDs1, NGC 6337, Sp 1) continue the trend, noted in Paper I, that soft $(\sim 0.6-1.0 \mathrm{keV}) \mathrm{X}$-ray sources likely indicate the presence of CSPN photospheric emission and/or wind shocks, while hard $(\gtrsim 1.0 \mathrm{keV}$ ) sources appear related to the presence of binary companions to CSPNe (Figure 4; R. Montez et al. 2014a, in preparation). Below are brief summaries of each of the Table 1 PNe with newly detected point-like X-ray emission.

HbDs1 is an irregularly elliptical nebula around the hot blue star LSS $1362\left(T_{\text {eff }}=116 \mathrm{kK}\right.$; Herald \& Bianchi 2011). 

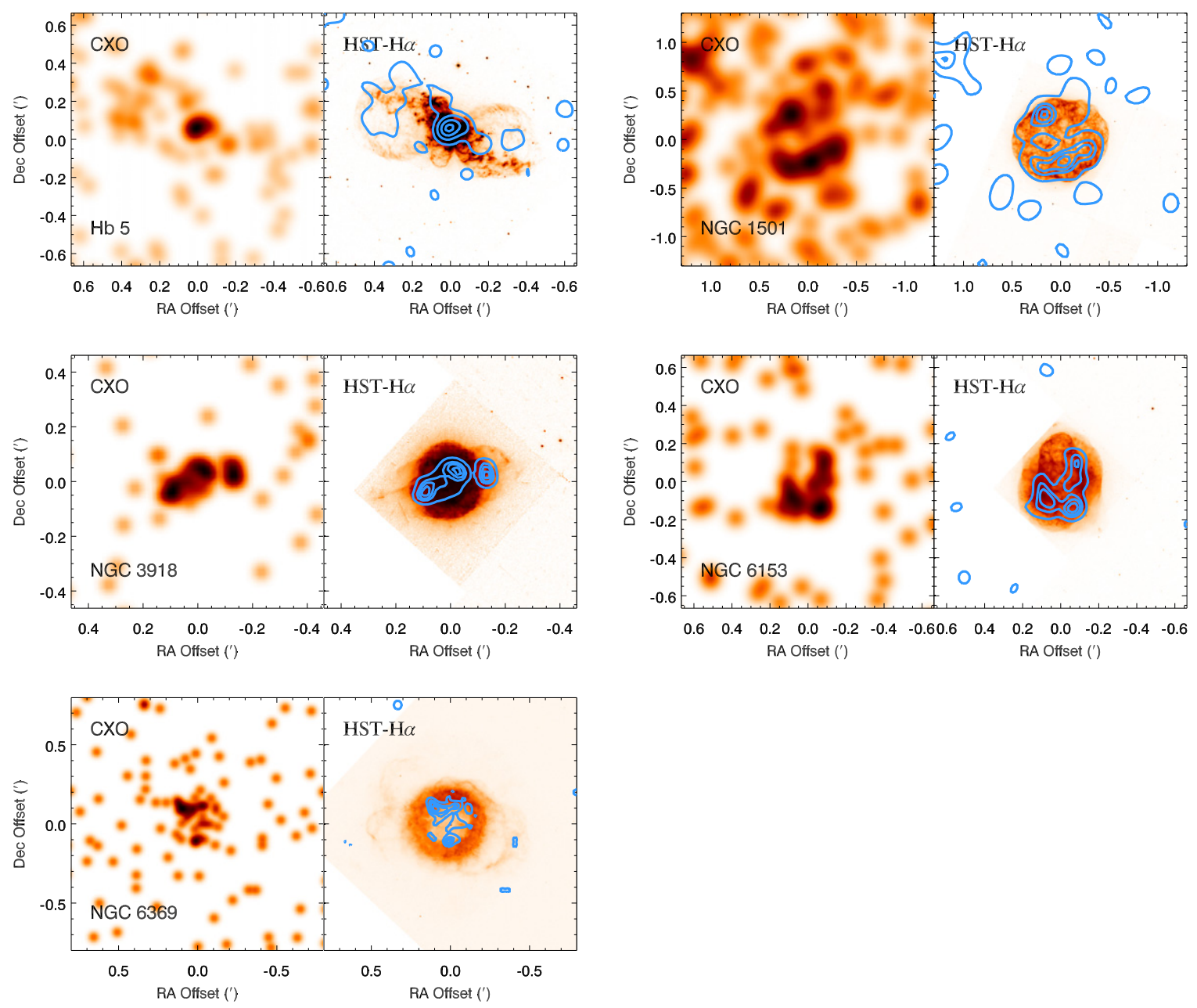

Figure 2. Images of Table $1 \mathrm{PNe}$ in which diffuse X-ray emission has been detected by Chandra. The left and right panel for each PN display, respectively, Chandra 0.5-1.2 keV images filtered to efficiently remove the soft background and Chandra contours overlaid on optical images. The Chandra images of Hb 5, NGC 1501, and NGC 6153 have been smoothed with a 12".5 FWHM Gaussian, while the Chandra images of NGC 3918 and NGC 6369 have been smoothed with a FWHM of 3". The contour levels of all but $\mathrm{Hb} 5$ are $30 \%, 60 \%, 75 \%$, and $90 \%$ of the maximum X-ray surface brightness (Hb 5 has contour levels of $10 \%, 30 \%, 60 \%$, and $90 \%$ ).

(A color version of this figure is available in the online journal.)
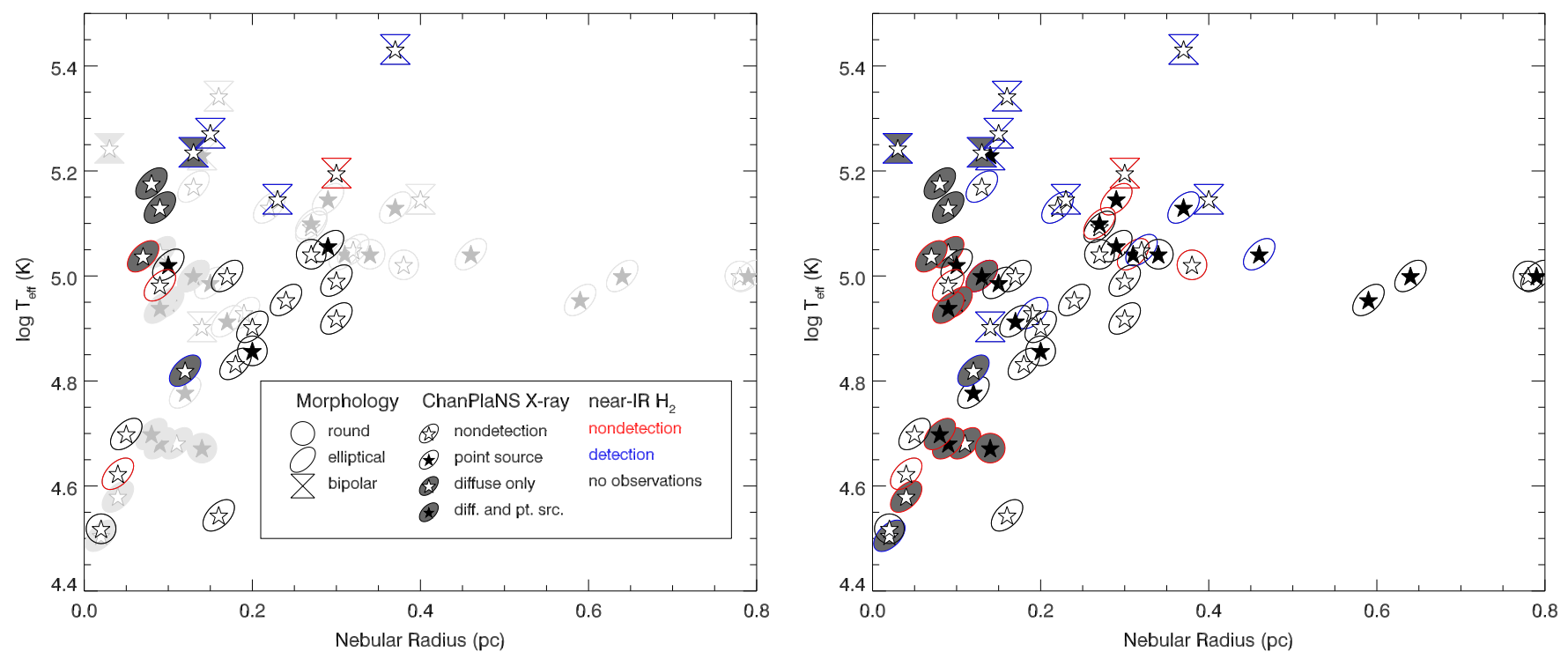

Figure 3. Plots of CSPN $T_{\text {eff }}$ vs. PN radius for all ChanPlaNS observed objects, with symbols indicating the presence or absence of diffuse or point-like X-ray emission, as well as PN morphology and the presence or absence of $\mathrm{H}_{2}$ emission. In the left panel, the highlighted objects represent Cycle 14 data and the light gray objects represent Cycle 12 and archival data (Paper I).

(A color version of this figure is available in the online journal.) 
Table 2

Log of Chandra Observations

\begin{tabular}{|c|c|c|c|}
\hline Name & OBSID & Date & $\begin{array}{c}\text { Exposure } \\
(\mathrm{ks})\end{array}$ \\
\hline A65 & 14583 & 2013 Aug 22 & 29.7 \\
\hline HaWe 13 & 14578 & 2013 Jul 15 & 18.6 \\
\hline $\mathrm{Hb} 5$ & 14596 & 2013 Oct 18 & 29.1 \\
\hline HbDs 1 & 14575 & 2013 Aug 10 & 19.5 \\
\hline He $2-11$ & 14581 & 2013 Aug 10 & 29.6 \\
\hline IC 1295 & 14585 & 2013 Aug 04 & 29.7 \\
\hline IC 2149 & 14593 & 2012 Dec 30 & 28.7 \\
\hline IC 4637 & 14586 & 2014 Mar 08 & 29.6 \\
\hline IC $5148 / 50$ & 14576 & 2013 Apr 07 & 19.7 \\
\hline M 1-26 & 14584 & 2013 Aug 10 & 29.6 \\
\hline M 1-41 & 14591 & 2013 Mar 02 & 29.6 \\
\hline NGC 1501 & 14574 & 2012 Dec 08 & 19.7 \\
\hline NGC 2899 & 14589 & 2013 May 28 & 29.6 \\
\hline NGC 3918 & 14597 & 2013 Jul 31 & 30.0 \\
\hline NGC 6026 & 14588 & 2013 Jun 29 & 29.6 \\
\hline NGC 6072 & 14590 & 2014 Jan 23 & 29.6 \\
\hline NGC 6153 & 14579 & 2014 Jan 17 & 28.6 \\
\hline NGC 6337 & 14577 & 2013 Aug 04 & 19.7 \\
\hline NGC 6369 & 14594 & 2013 Feb 18 & 29.6 \\
\hline NGC 6894 & 14587 & 2013 Dec 13 & 29.2 \\
\hline NGC 7076 & 14592 & 2013 Mar 29 & 29.6 \\
\hline NGC 7354 & 14595 & 2013 Dec 26 & 29.6 \\
\hline Sh $2-71$ & 14582 & 2013 Oct 07 & 29.6 \\
\hline Sp 1 & 14580 & 2013 Jan 24 & 29.4 \\
\hline
\end{tabular}

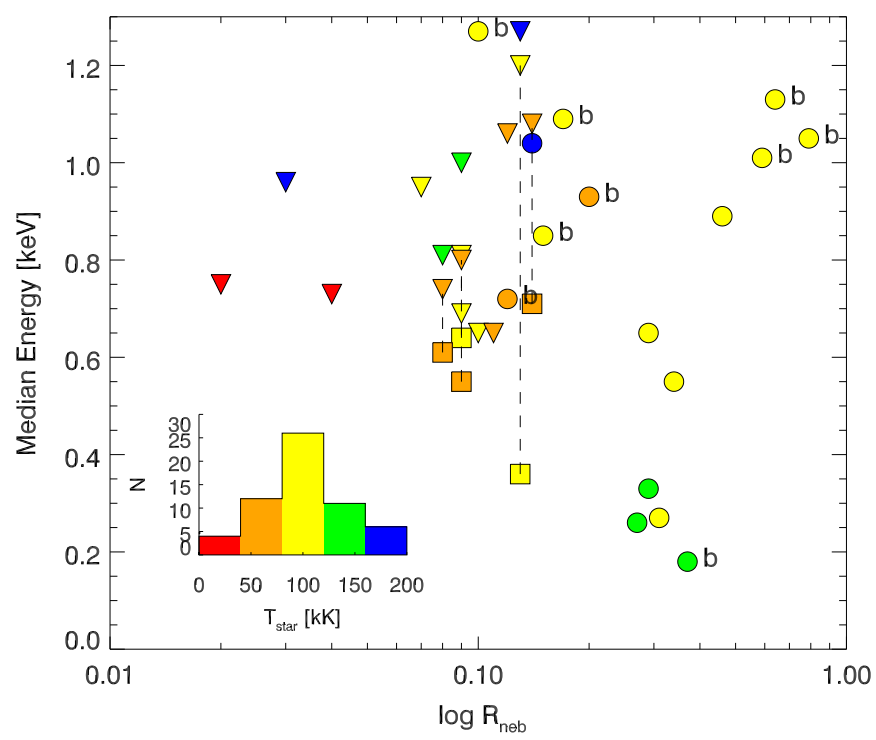

Figure 4. Median X-ray photon energy vs. nebular radius for all Cycle $12+14+$ archival sample PNe detected as X-ray sources. Symbol shapes indicate the nature of the PN X-ray source (triangles: diffuse sources; circles: CSPN point sources; squares: diffuse+CSPN sources) and are color-coded according to CSPN $T_{\text {eff }}$, whose distribution for the observed sample is shown in the inset histogram. Known binary CSPNe are indicated by "b." The dashed line connects the diffuse+CSPN emission with the corresponding diffuse emission for sources that exhibit both types of emission.

(A color version of this figure is available in the online journal.)

The nebula has enhanced $\mathrm{H} \alpha$ emission along its northern and southern limbs. The magnetic field properties of the CSPN have been debated. A kG field was inferred by Jordan et al. (2005, 2012) from spectropolarimetric data taken with the ESO Very Large Telescope; however, more recent FORS2 observations showed no evidence of such a strong field (with upper limits of $B<600 \mathrm{G}$; Leone et al. 2011; Bagnulo et al. 2012).
Table 3

Planetary Nebulae X-Ray Point Source Characteristics

\begin{tabular}{|c|c|c|c|c|}
\hline Name & $\begin{array}{c}N^{\mathrm{a}} \\
\text { (photons) }\end{array}$ & $\begin{array}{c}C^{\mathrm{b}} \\
\left(\mathrm{ks}^{-1}\right)\end{array}$ & $\begin{array}{c}\text { Median } E^{\mathrm{c}} \\
\quad(\mathrm{keV})\end{array}$ & $\begin{array}{c}E \text { Range }^{\mathrm{d}} \\
(\mathrm{keV})\end{array}$ \\
\hline $\mathrm{A} 65^{\mathrm{e}}$ & 1 & $0.02 \pm 0.05$ & 0.96 & $0.96-1.09$ \\
\hline HaWe 13 & $\ldots$ & $<0.21$ & $\ldots$ & $\ldots$ \\
\hline $\mathrm{Hb} 5^{\mathrm{f}}$ & 15: & $0.51 \pm 0.09$ & 0.94: & $0.81-1.03$ \\
\hline HbDs 1 & 10 & $0.49 \pm 0.06$ & 0.65 & $0.56-0.78$ \\
\hline He $2-11$ & $\ldots$ & $<0.14$ & $\ldots$ & $\ldots$ \\
\hline IC 1295 & $\ldots$ & $<0.13$ & $\ldots$ & $\ldots$ \\
\hline IC 2149 & $\ldots$ & $<0.14$ & $\ldots$ & $\ldots$ \\
\hline IC 4637 & $\ldots$ & $<0.14$ & $\ldots$ & $\ldots$ \\
\hline IC 5148/50 & $\ldots$ & $<0.20$ & $\ldots$ & $\ldots$ \\
\hline M 1-26 & $\cdots$ & $<0.15$ & $\ldots$ & $\ldots$ \\
\hline M 1-41 & $\ldots$ & $<0.14$ & $\ldots$ & $\ldots$ \\
\hline NGC $1501^{\mathrm{g}}$ & $\ldots$ & $<0.20$ & $\ldots$ & $\ldots$ \\
\hline NGC 2899 & $\ldots$ & $<0.14$ & $\ldots$ & $\ldots$ \\
\hline NGC $3918^{g}$ & 21: & $0.71 \pm 0.06$ & $0.85:$ & $0.66-1.03$ \\
\hline NGC $6026^{\mathrm{e}}$ & 1 & $0.05 \pm 0.08$ & 1.16 & $0.90-2.31$ \\
\hline NGC 6072 & $\ldots$ & $<0.14$ & $\ldots$ & $\ldots$ \\
\hline NGC $6153^{g}$ & $\ldots$ & $<0.14:$ & $\ldots$ & $\ldots$ \\
\hline NGC 6337 & 39 & $1.99 \pm 0.07$ & 1.27 & $1.03-1.73$ \\
\hline NGC $6369^{g}$ & 8: & $0.27 \pm 0.07:$ & 1.06: & $0.96-2.38$ \\
\hline NGC 6894 & $\ldots$ & $<0.14$ & $\cdots$ & $\ldots$ \\
\hline NGC 7076 & $\ldots$ & $<0.14$ & $\ldots$ & $\ldots$ \\
\hline NGC 7354 & $\ldots$ & $<0.14$ & $\cdots$ & $\cdots$ \\
\hline Sh 2-71 & $\ldots$ & $<0.14$ & $\ldots$ & $\ldots$ \\
\hline Sp 1 & 11 & $0.37 \pm 0.07$ & 0.93 & $0.83-1.33$ \\
\hline
\end{tabular}

Notes.

a Number of source photons, after background subtraction.

b Source photon count rate.

c Median source photon energy.

${ }^{\mathrm{d}}$ Source photon energy range (25th through 75 th percentiles).

e Uncertain point source detections at the positions of known binaries. See Section 3.3.

$\mathrm{f}$ There is no point source apparent within $\mathrm{Hb} 5$.

g Point source counts, count rate (or upper limit), median energy, and energy ranges are uncertain due to presence of diffuse emission component.

Table 4

Planetary Nebulae: Chandra X-Ray Detection Statistics

\begin{tabular}{lccc}
\hline \hline Category $^{\mathrm{a}}$ & $N^{\mathrm{b}}$ & $N_{P X}{ }^{\mathrm{c}}$ & $N_{D X}{ }^{\mathrm{c}}$ \\
\hline Entire sample & 59 & $21(36 \%)$ & $16(27 \%)$ \\
\hline Round/elliptical, F08 & 47 & $19(40 \%)$ & $14(30 \%)$ \\
Bipolar, F08 & 12 & $2(17 \%)$ & $2(17 \%)$ \\
\hline Round/elliptical/irregular, SMV11 & 37 & $14(38 \%)$ & $9(24 \%)$ \\
Bipolar/multipolar, SMV11 & 22 & $7(32 \%)$ & $7(32 \%)$ \\
\hline Near-IR H not detected $_{\text {Near-IR H }}$ detected & 19 & $9(47 \%)$ & $10(53 \%)$ \\
\hline Known binary CSPN & 18 & $3(17 \%)$ & $4(22 \%)$ \\
\hline
\end{tabular}

Notes.

a Morphologies as listed and defined in Column 3 of Table 1 and associated footnotes; CSPN binary detections and $\mathrm{H}_{2}$ detections as listed, respectively, in Columns 9 and 10 of Table 1.

b Total number of sample PNe in each category.

${ }^{c}$ Number of PNe in each category displaying point-like and/or diffuse X-ray emission in Chandra imaging (Paper I).

The detected X-ray point source has a median energy of $0.65 \mathrm{keV}$, which may indicate self-shocking within the stellar winds previously detected at the CSPN (Herald \& Bianchi 2011; Guerrero \& De Marco 2013), possibly combined with photospheric emission (see Montez \& Kastner 2013). 

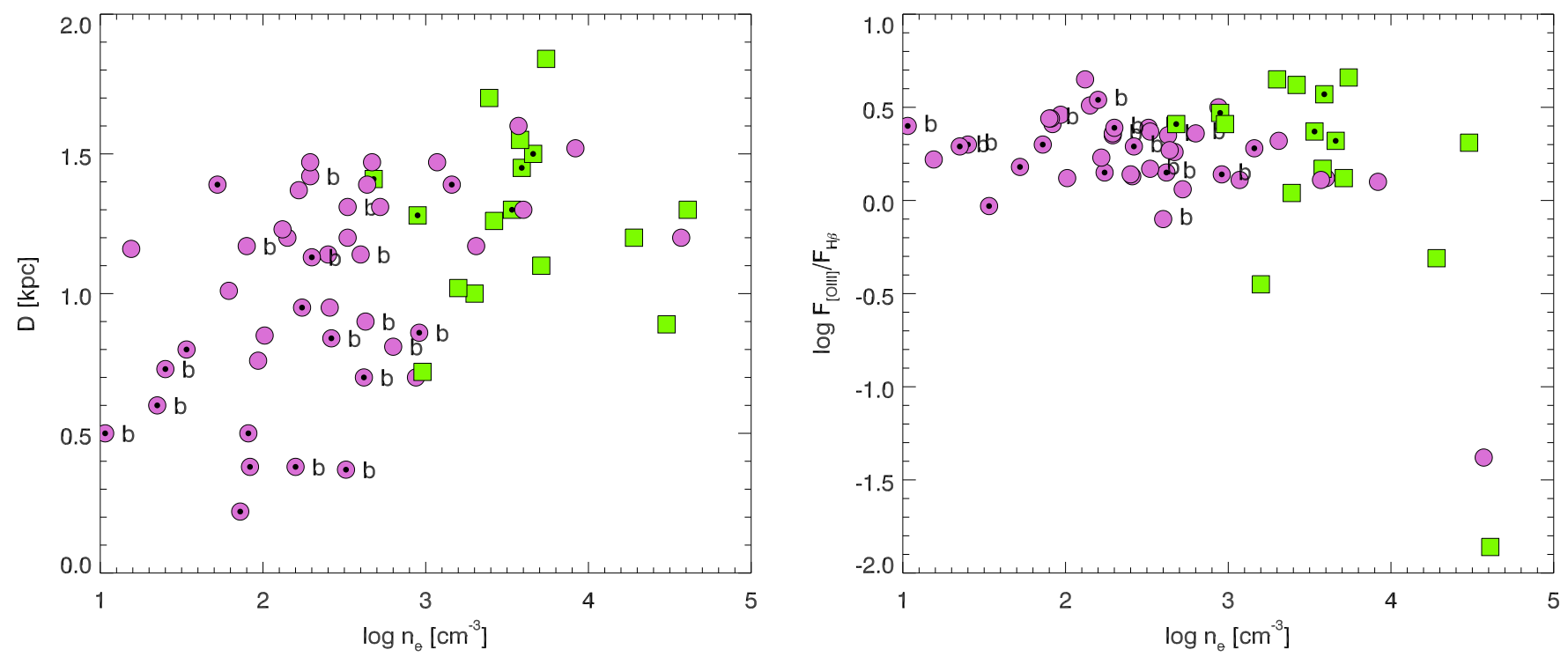

Figure 5. Distance (left) and ratio of [O III] to $\mathrm{H} \beta$ fluxes (right) vs. the $\log$ of electron density $n_{e}$ for PNe detected (green squares) and not detected (purple circles) as ChanPlaNS diffuse X-ray sources. A bullet within each symbol indicates that the CSPNe was detected as a point-like X-ray source. A "b" next to a symbol indicates the CSPN is a known binary.

(A color version of this figure is available in the online journal.)

NGC 6337 is seen as a ring nebula with radial filaments. This PN harbors a nearly face-on $\left(i \lesssim 15^{\circ}\right)$ close binary $(0.17$ day period) central star system (Hillwig et al. 2010). It is likely that the nebula is viewed nearly pole-on as well, suggesting a bipolar structure with a narrow waist around the binary (García-Díaz et al. 2009). The close binary is most likely the product of a common envelope phase (De Marco et al. 2011), leaving behind a pre-WD and a cool main-sequence star (Hillwig et al. 2010). The X-ray point source associated with this binary has a median energy of $1.27 \mathrm{keV}$, the hardest emission detected from a point source in the ChanPlaNS sample thus far. This hard X-ray detection provides strong support for a model in which the X-rays originate from a cool, coronally active companion (see Section 4).

Sp 1 appears similar to NGC 6337; this PN is also a diffuse ring on the plane of sky and harbors a close binary (2.9 day period) at its center (Bond \& Livio 1990; Jones et al. 2012; Bodman et al. 2012). Spatiokinematical modeling of the nebular shell shows that the nebula is a nearly pole-on hourglass shape (Jones et al. 2012). This is borne out by modeling of the CSPN by Bodman et al. (2012), which indicates a G0 or F8 main sequence companion with an orbital inclination of $10^{\circ}<i<20^{\circ}$, consistent with the generation of a near pole-on nebula. UV spectra of the CSPN show evidence for stellar winds (Patriarchi \& Perinotto 1991), which could be at best partly responsible for the X-ray point source at the CSPN. However, the median energy for the X-rays from the Sp 1 CSPN is $0.93 \mathrm{keV}$, making it one of the harder X-ray sources in the CHANPLANS sample, and suggesting that the companion dominates the detected emission.

\subsection{X-Ray Non-detections}

Table 3 lists the point source $X$-ray characteristics (net counts, count rates, median photon energies, and photon energy ranges) of each PN in the Cycle 14 sample. To obtain these results, the $0.3-3.0 \mathrm{keV}$ background counts were extracted from a sourcefree region near the central star. These background counts were scaled to the point source extraction radius of 3 '.5, and then subtracted from the counts of the same extraction region centered on the CSPN, to obtain the net source counts (or source count upper limits). Sources with less than six net counts are classified as undetected. Two sources lie below this backgroundsubtracted limit, A65 and NGC 6026, but could be considered as tentative detections; there are $\sim 4$ (source+background) counts found near their CSPNe, both of which have companion stars. There are a few sources above the net count limit that we

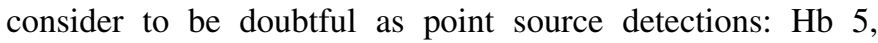
NGC 3918, and NGC 6369. Contamination from the diffuse X-ray emission in NGC 3918 makes it difficult to determine the contribution from a possible point source at the CSPN in the circular source region. NGC 6369 is a luminous, albeit reddened, PN that lies near the limit of our volume-limited sample, at a distance of $1.55 \mathrm{kpc}$ (Monteiro et al. 2004). Additionally, the diffuse X-ray emission of NGC 6369 is not concentrated at the CSPNe, which makes it unlikely to contaminate a point source detection. Finally, as noted in Section 3.2, $\mathrm{Hb} 5$ appears to have no point-like emission within the CSPN extraction region.

Several PNe that were undetected appear to fit within morphology trends typical of previous CHANPLANS point source and diffuse X-ray detections. IC 5148/50 is undetected, yet it has a morphology similar to Sp 1, suggesting that it may be a pole-on hourglass and, hence, could be the product of a binary system. In optical images, NGC 6894 has the multiple-shell structure of a hot bubble from which we would expect to see diffuse X-ray emission, though it is important to note that the central star is faint and on the WD cooling track. Similarly, NGC 7354 hosts multiple elliptical shells and ansae, similar to NGC 3918 and NGC 6153; however, no X-ray photons were detected from the innermost bubble of NGC 7354. These nondetections appear to place stringent limits on the timescales for $\mathrm{X}$-ray emitting hot bubbles within $\mathrm{PNe}$ (Section 4.2)—although we note that NGC 7354 is quite heavily reddened and, hence, any soft X-rays from its interior might suffer considerable absorption.

The objects with no detectable X-ray emission have varying morphologies (round, elliptical, bipolar), with at least half exhibiting inherent asymmetries. IC 4637 is an example of a PNe that falls within the range of nebular properties for the diffuse X-ray detections (Paper I), but was not detected. Specifically, 
IC 4637 has a radius of $0.05 \mathrm{pc}$ and elliptical morphology (Frew 2008). Additionally, IC 2149 is a young, compact bipolar nebula with an elliptical central shell and may be the product of a lowmass progenitor and/or binary evolution (Vázquez et al. 2002). Upper limit estimates for X-ray luminosity $L_{X}$ for each of these sources (R. Montez et al. 2014, in preparation) will help place constraints on the sizes, plasma densities, and morphologies of $\mathrm{X}$-ray emitting nebulae.

\section{DISCUSSION}

The sample of $59 \mathrm{PNe}$ within $\sim 1.5 \mathrm{kpc}$ observed to date by Chandra constitutes a representative set of X-ray observations of mostly compact and, hence, predominately relatively young PNe within the solar neighborhood. Adding the $24 \mathrm{PNe}$ observed in Cycle 14 to the initial sample (see Paper I) reduces the overall PN X-ray detection rate from $\sim 70 \%$ to $\sim 54 \%$ $(32 / 59)$. This decrease most likely reflects the composition of the initial CHANPLANS sample; these objects were chosen based on the presence of bright excitation lines indicative of hot CSPNe $\left(T_{\text {eff }} \gtrsim 10^{5} \mathrm{~K}\right)$ and/or structure that resulted from rapid evolution (Paper I), while the Cycle 14 objects were chosen to complete the X-ray census of compact $\left(R_{\text {neb }} \lesssim 0.4 \mathrm{pc}\right) \mathrm{PNe}$ within $\sim 1.5 \mathrm{kpc}$. Nevertheless, the Cycle 14 results underscore trends apparent in the X-ray properties of PNe, as we discuss in the following subsections.

\subsection{Diffuse X-Ray Emission from [WR]-type CSPNe}

The CSPNe that are H-deficient and display spectroscopic evidence for fast stellar winds and high mass-loss rates (up to $10^{-6} M_{\odot} \mathrm{yr}^{-1}$; Crowther et al. 1998; DePew et al. 2011) are classified as [WR]-type because of their similarity, as a class, to luminous WR stars. The primary difference between the two classes is that the [WR]-type stars descend from intermediate mass $\left(1-8 M_{\odot}\right)$ stars, whereas "classical" WR stars are the descendants of massive ( $>25-30 M_{\odot}$ ) stars (Crowther et al. 2006). In total, there are five known [WR]-type CSPNe in the ChanPlaNS sample: NGC 1501 and NGC 6369 from Cycle 14 (Table 1), as well as NGC 40, NGC 2371, and BD +30 3639 from Cycle 12 and archival observations (Paper I). All five of these [WR]-type CSPN objects display diffuse X-ray emission. The only consistent morphological similarity among all of the [WR] type CSPNe is that their main bubbles appear elliptical (Frew 2008). In each case, the diffuse X-ray emission is enclosed by the central optical bubble, although the morphology of the $\mathrm{X}$-ray emission varies considerably from object to object.

$B D+30^{\circ} 3639$ : The unusually bright, diffuse emission fills the central bubble, perhaps as a consequence of its very young age $\left(\sim 10^{3}\right.$ yr; Frew 2008).

$N G C$ 40: The emission appears within a partial ring that traces the inner edge of the bright optical rim of the nebula (Montez et al. 2005).

$N G C$ 2371: The diffuse emission is contained within the bright nebular rim but does not seem to follow any significant optical feature, and is highly asymmetric.

$N G C$ 1501: The X-ray emission traces the rim of the nebula, echoing the limb-brightened morphology of WR star windblown bubble S308 (Toalá et al. 2012).

NGC 6369: The diffuse emission is asymmetrical and strongest along the northern limb of the central bubble.

The X-ray results for most of the above PNe, with the possible exception of NGC 2371, reveal some potential similarities between $\mathrm{PNe}$ with [WR]-type CSPNe and the wind-blown bubbles of massive WR stars. The X-ray limb-brightening in massive WR star wind-blown bubbles, such as S308, is the result of the hot WR wind cooling as it comes in contact with the previously ejected red (or yellow) supergiant or luminous blue variable material (García-Segura et al. 1996a, 1996b; Freyer et al. 2003; Toalá \& Arthur 2011; Toalá et al. 2012; Dwarkadas \& Rosenberg 2013). A similar process occurs in $\mathrm{PNe}$, wherein the X-ray emission is contained within the ejected AGB envelope and does not come in direct contact with the ISM. The question remains: is the diffuse X-ray emission from [WR] CSPNe limb-brightened or not? Given the photon-starved nature of our detections, we will require Poisson-based models for comparison with the observations to adequately answer this question.

\subsection{Diffuse X-Ray Emission and PN Size, Age, and Electron Density}

The diffuse X-ray emission from hot bubbles in $\mathrm{PNe}$ is evidently confined to early phases in PN evolution. In Paper I, we pointed out that the central bubbles of PNe with diffuse emission tend to have elliptical, nested-shell morphologies and radii $\lesssim 0.15 \mathrm{pc}$ (Figure 3 and Tables 1 and 4 ). This radius limit corresponds to dynamical ages $\lesssim 5 \times 10^{3} \mathrm{yr}$, roughly a quarter of the $(21 \pm 5) \times 10^{3} \mathrm{yr}$ mean visibility time of PNe (Jacob et al. 2013). Our Cycle 14 detections and non-detections thereby reinforce the inference, made in Paper I, that diffuse X-ray emission is restricted to the most compact nebulae. We note that the correlation of diffuse X-rays with elliptical morphology is ambiguous (Table 4), but it remains the case that PNe with closed structures (whether bubbles or lobes) are more likely to be detected as diffuse X-ray sources.

Furthermore, from Figure 5 we see that with the exception of NGC 2371, all ChanPlaNS PNe with diffuse X-ray emission have nebular densities $n_{e} \gtrsim 1000 \mathrm{~cm}^{-3}$, as determined via their $\mathrm{H} \alpha$ line luminosities and nebular radii, which are in turn determined from the $\mathrm{H} \alpha$ surface brightness versus radius relation (Frew 2008; Frew et al. 2014b). In contrast, the diffuse X-ray detection rate is essentially independent of distance and nebular excitation. Since PN density and radius are correlated, the remarkably sharp $n_{e}$ boundary between diffuse X-ray detections and non-detections in Figure 5 further underscores the notion of a limit on the timescale for energetic (nebula-shaping) wind interactions in PNe. Moreover, Figure 5 suggests that, from the spectroscopy of density-sensitive lines (e.g., [O II], [S II]), it is possible to ascertain whether a PN might currently be in a wind-collision phase, when we would expect to see diffuse $\mathrm{X}$-ray emission. Nevertheless, the duration of the windinteraction phase is a complex problem that must account for (1) luminosity of the central star (e.g., Hb 5 high, NGC 5148 low), (2) expected hydrogen column density, and (3) spectral type of the central star (i.e., whether it is [WR] or not).

This density limit may explain the X-ray non-detection of nebulae like NGC 6894 and NGC 6804 (Paper I), which appear morphologically similar to known diffuse X-ray emitters. NGC 6894 has a nebular radius of 0.17 pc and NGC 6804 has a nebular radius of $0.19 \mathrm{pc}$, and both have a density $\log n_{e} \sim 2.7$. Thus, evidently, both NGC 6894 and NGC 6804 lie close to, but just below, the $\log n_{e} \mathrm{X}$-ray detection threshold. It is important to keep in mind that electron density is not a good indicator of PN evolutionary state. Fast evolving stars reach low luminosities at relatively high nebular densities, but remain undetected in diffuse X-rays (Steffen et al. 2008). Upper limit estimates and observations of additional nebulae similar to these-with $n_{e} \gtrsim$ 
$1000 \mathrm{~cm}^{-3}$ and closed optical morphologies (multiple shells, well-defined inner bubbles) —will allow us to answer questions about diffuse emission such as the following. What is the lifetime of the hot bubble shaping phase? How does it relate to the mass of the CSPN progenitor? What is the relationship between diffuse X-ray emission and the final mass of the core, i.e., the WD remnant? We aim to revisit the question of diffuse X-ray dependence on age in R. Montez et al. (2014, in preparation; also see Schönberner et al. 2014).

\subsection{Binary Detections/Non-detections}

Six additional known binary CSPNe were observed in Cycle 14, all of which are close binaries ( $p<3$ days). Two of these six, NGC 6337 and Sp 1, had detectable point source $\mathrm{X}$-ray emission. Both PNe appear to have nearly pole-on hourglass structures. The median energy for both point sources is relatively hard ( $\gtrsim 1.0 \mathrm{keV})$, which implies that the CSPN X-ray emission is due to late-type companions that display elevated levels of coronal activity. These binary, X-ray-luminous CSPNe are therefore similar to the CSPNe in DS 1, HFG 1, and LoTr 5, which are thought to be systems in which mass transfer from PN progenitor to late-type companion has spun up the companion (Jeffries \& Stevens 1996; Soker \& Kastner 2002; Montez et al. 2010); alternatively, in many or most of these cases of $\mathrm{X}$-ray-emitting binary CSPNe, it is possible the coronal activity of the companion is due to tidal locking that has resulted in rapid, synchronous rotation (a possibility we further explore in Montez et al. 2014). Both NGC 6337 and Sp 1 represent the two most compact (and therefore youngest) PNe harboring X-ray point sources at known binary CSPNe in the CHANPLANS sample (Figure 4). The periods of the close binaries within A65 (1.00 day; Bond \& Livio 1990; Shimansky et al. 2009; T. Hillwig et al. 2014, in preparation), He 2-11 (0.61 days; Jones et al. 2014b), and NGC 6026 (0.53 days; Hillwig et al. 2010) lie between those of NGC 6337 (0.17 days; Hillwig et al. 2010) and Sp 1 (2.91 days; Bond \& Livio 1990), but no point source X-ray emission was detected from the former three PNe. This leaves us with unanswered questions: What is the relationship between binary period/separation and X-ray point source emission? What are the characteristics of secondaries within binaries that do exhibit X-ray emission?

IC 5148/50 is a potentially significant non-detection, given its morphological similarity to $\mathrm{Sp} 1$. Based on imagery alone, Sp 1 appears to have a spherical morphology, but spatiokinematical modeling reveals this is a projection effect and its intrinsic structure is that of a pole-on hourglass (Jones et al. 2012). Such bipolar structures are likely to be the products of binary interactions (see, e.g., Balick \& Frank 2002, and references therein). The non-detection of IC 5148/50 may indicate that while it is morphologically similar to Sp 1, structurally it may be quite different. From $\mathrm{H} \alpha,[\mathrm{N}$ II] , and [O III] observations (Hua et al. 1998), it is clear that IC 5148/50 hosts not only internal elliptical structure but also a helical structure. Observations of $\mathrm{H}_{2}$ (Kastner et al. 1996) and/or spatially resolved, high-resolution emission line spectroscopy (for use in spatiokinematical modeling) are necessary to determine whether or not IC $5148 / 50$ has inherently bipolar versus elliptical structure. The CSPN of IC 5148/50 also has no infrared excess indicative of an unresolved cool companion (De Marco et al. 2013; Douchin et al. 2014).

It is possible that many other relatively X-ray-luminous CSPN have gone undetected in our sample of PNe. From Figure 5 we see that the fraction of PNe with detected point- like X-ray emission is strongly dependent on distance. All six sample PNe within $\lesssim 0.6 \mathrm{kpc}$ have point-like emission, whereas beyond $1.3 \mathrm{kpc}$ the detection fraction drops to $\sim 35 \%$. This drop in detection fraction is likely the result of increased interstellar extinction obscuring the softer X-ray point sources, and furthermore implies that we lack the sensitivity necessary to detect even the (harder) binary CSPNe at distances much beyond $\sim 1 \mathrm{kpc}$. Further discussion concerning the implications of the distance dependence of the X-ray detection rate of CSPNe can be found in Montez et al. (2014).

\subsection{Diffuse X-Ray Emission from Jet-Wind Interactions During Common Envelope Ejection?}

Several elliptical PNe display diffuse X-ray emission morphologies wherein the elongated diffuse emission appears to include peaks on two opposite locations along the long axis of the elliptical rim. Examples are NGC 3242, NGC 6543, NGC 7009, NGC 7662 (Paper I), and NGC 3918 (Figure 1). One possible interpretation of such an X-ray morphology, with far-reaching implications, is that the diffuse emission emanates from gas heated by shocks as low-mass jets with velocities of a few $100 \mathrm{~km} \mathrm{~s}^{-1}$ passed through material previously ejected in a common envelope interaction (Akashi et al. 2008). Such post-common envelope jets would most likely be launched by an accretion disk around a main sequence companion to the PN progenitor star during the progenitor's post-AGB phase. Indeed, it is possible that such jets are the "last gasps" of outflows launched during the final stages of common envelope evolution (Tocknell et al. 2014). If so, then the diffuse X-ray emission from elliptical PNe might hint at the operation of jets in the final removal of common envelopes formed by evolved, close binary systems. The X-ray emission morphologies of elliptical PNe, and their potential implications for common envelope models of PN formation, will be further explored in subsequence papers in the ChanPlaNS series (e.g., R. Montez et al. 2014, in preparation).

\section{SUMMARY}

We present results from the most recent set of observations obtained by the Chandra Planetary Nebula Survey (CHANPLANS), the first comprehensive X-ray survey of PNe in the solar neighborhood (within $\sim 1.5 \mathrm{kpc}$ ). The survey is designed to place constraints on the frequency of the appearance and range of $\mathrm{X}$-ray spectral characteristics of X-ray-emitting PN central stars and the evolutionary timescales of wind-shock-heated bubbles within PNe. CHANPLANS began with a combined Cycle 12 and archive Chandra survey of 35 PNe. CHANPLANS continued via a Chandra Cycle 14 Large Program which targeted 24 additional compact $\left(R_{\text {neb }} \lesssim 0.4 \mathrm{pc}\right.$ ), young PNe that lie within $\sim 1.5 \mathrm{kpc}$. The highlights of the CHANPLANS Cycle 14 results include the following.

1. The overall X-ray detection rate for relatively compact ( $\lesssim 0.4 \mathrm{pc}$ ) PNe within $\sim 1.5 \mathrm{kpc}$ observed thus far by Chandra is $\sim 54 \%$.

2. Nearly $40 \%$ of the ChanPlaNS sample PNe host X-rayluminous point sources at their CSPNe. This includes three new detections of CSPNe X-ray sources within the Cycle 14 sample PNe.

3. Roughly $60 \%$ of the known binaries within the CHANPLANS sample display point-like X-ray emission, including firsttime Cycle 14 detections of X-rays from the CSPNe of NGC 6337 and Sp 1. 
4. All PNe with [WR]-type CSPN within the CHAnPlaNS sample observed thus far $\left(\mathrm{BD}+30^{\circ} 3639\right.$, NGC 40, NGC 2371, NGC 1501, NGC 6369) display diffuse X-ray emission. With the exception of NGC 2371, all of these $\mathrm{PNe}$ have elliptical morphologies. In each case, the diffuse $\mathrm{X}$-ray emission resembles the limb-brightened morphology of wind-blown bubbles blown by massive WR stars.

5. The addition of the Cycle 14 results brings the overall ChanPlaNS diffuse X-ray detection rate to $\sim 27 \%$.

6 . It has become clearer that diffuse X-ray emission is associated with young $\left(\lesssim 5 \times 10^{3} \mathrm{yr}\right)$ PNe with compact $\left(R_{\text {neb }} \lesssim 0.15 \mathrm{pc}\right)$, closed structures and high central electron densities $\left(n_{e} \gtrsim 1000 \mathrm{~cm}^{-3}\right)$. Typically, such nebulae display nested-shell elliptical morphologies and rarely show $\mathrm{H}_{2}$ emission and/or pronounced butterfly structures. $\mathrm{Hb} 5$ is a notable exception.

7. The ChanPlanS detection rate of diffuse X-ray emission from PNe within $\sim 1.5 \mathrm{kpc}$ appears largely independent of distance and excitation, if the latter is measured via the flux ratio of $[\mathrm{O} \mathrm{III}]$ and $\mathrm{H} \beta$ lines.

8. In contrast, the CHANPLANS detection rate of point-like $\mathrm{X}$-ray emission from PNe within $\sim 1.5 \mathrm{kpc}$ appears strongly dependent on distance, reflecting the relatively low intrinsic $\mathrm{X}$-ray luminosities of CSPNe combined with the effects of interstellar extinction.

We note that in addition to the need to acquire data for a larger sample of PNe-particularly high- $n_{e} \mathrm{PNe}$ and those harboring known binary central stars-we must carry out estimations of upper limits on $L_{X}$ in order to reaffirm our claims and to properly answer our open questions. Further analysis of these and future CHANPLANS data and results describing both point-like X-ray emission from CSPNe (Montez et al. 2014) and diffuse X-ray emission from PNe (R. Montez et al. 2014, in preparation) will continue to refine models of PN shaping mechanisms, X-ray emission timescales, and the role of binarity in PN formation and evolution.

We thank the anonymous referee for helpful comments. This research was supported via award No. GO3-14019A to RIT issued by the Chandra X-ray Observatory Center, which is operated by the Smithsonian Astrophysical Observatory for and on behalf of NASA under contract NAS8-03060. Jesús A. Toalá and Martín A. Guerrero are supported by the Spanish MICINN grant AYA 2011-29754-C03-02 co-funded with FEDER funds. The Digitized Sky Surveys were produced at STScI under U.S. Government Grant NAG W-2166. This research has also made use of the SIMBAD database, operated at CDS, Strasbourg, France.

\section{REFERENCES}

Akashi, M., Meiron, Y., \& Soker, N. 2008, NewA, 13, 563

Bagnulo, S., Landstreet, J. D., Fossati, L., \& Kochukhov, O. 2012, A\&A, 538, 22

Balick, B., \& Frank, A. 2002, ARA\&A, 40, 439

Bernard-Salas, J., \& Tielens, A. G. G. M. 2005, A\&A, 431, 523

Bilíková, J., Chu, Y.-H., Gruendl, R. A., \& Maddox, L. A. 2010, AJ, 140, 1433

Bilíková, J., Chu, Y.-H., Gruendl, R. A., Su, K. Y. L., \& De Marco, O. 2012, ApJS, 200, 3

Bodman, E. H. L., Schaub, S. C., \& Hillwig, T. 2012, JSARA, 5, 19

Boffin, H. M. J., Miszalski, B., Rauch, T., et al. 2012, Sci, 338, 773

Bohigas, J. 2001, RMxAA, 37, 237

Bond, H. E., \& Livio, M. 1990, ApJ, 355, 568

Cassinelli, J. P., Cohen, D. H., Macfarlane, J. J., Sanders, W. T., \& Welsh, B. Y. 1994, ApJ, 421, 705
Clegg, R. E. S., Harrington, J. P., Barlow, M. J., \& Walsh, J. R. 1987, ApJ, 314,551

Corradi, R. L. M., Perinotto, M., Villaver, E., Mampaso, A., \& Gonçalves, D. R. 1999, ApJ, 523, 721

Corradi, R. L. M., Sabin, L., Miszalski, B., et al. 2011, MNRAS, 410, 1349

Crowther, P. A., De Marco, O., \& Barlow, M. J. 1998, MNRAS, 296, 367

Crowther, P. A., Morris, P. W., \& Smith, J. D. 2006, ApJ, 636, 1033

De Marco, O. 2009, PASP, 121, 316

De Marco, O., Passy, J.-C., Frew, D. J., Moe, M., \& Jacoby, G. H. 2013, MNRAS, 428, 2118

De Marco, O., Passy, J. C., Moe, M., et al. 2011, MNRAS, 411, 2277

DePew, K., Parker, Q. A., Miszalski, B., et al. 2011, MNRAS, 414, 2812

Douchin, D., De Marco, O., Frew, D. J., et al. 2014, MNRAS, submitted

Drew, J. E., Gonzalez-Solares, E., Greimel, R., et al. 2014, MNRAS, 440, 2036

Dwarkadas, V. V., \& Rosenberg, D. L. 2013, HEDP, 9, 226

Ercolano, B., Morisset, C., Barlow, M. J., Storey, P. J., \& Liu, X. W. 2003, MNRAS, 340, 1153

Ercolano, B., Wesson, R., Zhang, Y., et al. 2004, MNRAS, 354, 558

Frew, D. J. 2008, PhD thesis, Macquarie Univ.

Frew, D. J., Bojičić, I. S., Parker, Q. A., et al. 2014a, MNRAS, 440, 1080

Frew, D. J., Parker, Q. A., \& Bojičić, I. S. 2014b, MNRAS, submitted

Freyer, T., Hensler, G., \& Yorke, H. W. 2003, ApJ, 594, 888

García-Díaz, M. T., Clark, D. M., López, J. A., Steffen, W., \& Richer, M. G. 2009, ApJ, 699, 1633

García-Segura, G., Langer, N., \& Mac Low, M. M. 1996a, A\&A, 316, 133

García-Segura, G., Mac Low, M. M., \& Langer, N. 1996b, A\&A, 305, 229

Guerrero, M. A., \& De Marco, O. 2013, A\&A, 553, A126

Guerrero, M. A., Toalá, J. A., Medina, J. J., et al. 2013, A\&A, 557, A121

Herald, J. E., \& Bianchi, L. 2011, MNRAS, 417, 2440

Hillwig, T. C., Bond, H. E., Afşar, M., \& De Marco, O. 2010, AJ, 140, 319

Hillwig, T. C., Frew, D. J., Louie, M., et al. 2014, AJ, submitted

Hua, C. T., Dopita, M. A., \& Martinis, J. 1998, A\&AS, 133, 361

Huckvale, L., Prouse, B., Jones, D., et al. 2013, MNRAS, 434, 1505

Jacob, R., Schönberner, D., \& Steffen, M. 2013, A\&A, 558, A78

Jeffries, R. D., \& Stevens, I. R. 1996, MNRAS, 279, 180

Jones, D., Boffin, H. M. J., Miszalski, B., et al. 2014a, A\&A, 562, A89

Jones, D., Mitchell, D. L., Lloyd, M., et al. 2012, MNRAS, 420, 2271

Jones, D., Santander-Garcia, M., Boffin, H. M. J., Miszalski, B., \& Corradi, R. L. M. 2014b, in Asymmetrical Planetary Nebulae VI conference, ed. C. Morisset, G. Delgado Inglada, \& S. Torres Peimbert, http://www.astroscu.unam.mx/apn6/PROCEEDINGS/ (arXiv:1401.4119)

Jordan, S., Bagnulo, S., Werner, K., \& O’Toole, S. J. 2012, A\&A, 542, A64

Jordan, S., Werner, K., \& O’Toole, S. J. 2005, A\&A, 432, 273

Kastner, J. H., Montez, R., Jr., Balick, B., \& De Marco, O. 2008, ApJ, 672, 957

Kastner, J. H., Montez, R., Jr., Balick, B., et al. 2012, AJ, 144, 58

Kastner, J. H., Weintraub, D. A., Gately, I., Merrill, K. M., \& Probst, R. G. 1996, ApJ, 462, 777

Kwok, S., Purton, C. R., \& Fitzgerald, P. M. 1978, ApJL, 219, L125

Leone, F., Martínez González, M. J., Corradi, R. L. M., Privitera, G., \& Manso Sainz, R. 2011, ApJL, 731, L33

Li, J., Kastner, J. H., Prigozhin, G. Y., et al. 2004, ApJ, 610, 1204

López, J. A., García-Díaz, M. T., Steffen, M., Riesgo, H., \& Richer, M. G. 2012, ApJ, 750, 131

Marten, H., \& Schönberner, D. 1991, A\&A, 248, 590

Miszalski, B., Corradi, R. L. M., Boffin, H. M. J., et al. 2011, MNRAS, 413, 1264

Monteiro, H., Schwarz, H. E., Gruenwald, R., \& Heathcote, S. 2004, ApJ, 609, 194

Montez, R., Jr., De Marco, O., Kastner, J. H., \& Chu, Y.-H. 2010, ApJ, 721,1820

Montez, R., Jr., \& Kastner, J. H. 2013, ApJ, 766, 26

Montez, R., Jr., Kastner, J. H., Balick, B., et al. 2014, ApJ, submitted

Montez, R., Jr., Kastner, J. H., Balick, B., \& Frank, A. 2009, ApJ, 694, 1481

Montez, R., Jr., Kastner, J. H., De Marco, O., \& Soker, N. 2005, ApJ, 635, 381

Nordhaus, J., Blackman, E. G., \& Frank, A. 2007, MNRAS, 376, 599

Parker, Q. A., Phillipps, S., Pierce, M. J., et al. 2005, MNRAS, 362, 689

Patriarchi, P., \& Perinotto, M. 1991, A\&A, 91, 325

Peña, M., Rechy-García, J. S., \& García-Rojas, J. 2013, RMxAA, 49, 87

Perinotto, M., Schönberner, D., Steffen, M., \& Calonaci, C. 2004, A\&A, 414, 993

Pottasch, S. R., \& Bernard-Salas, J. 2010, A\&A, 517, A95

Ramos-Larios, G., Guerrero, M. A., Vazquez, R., \& Phillips, J. P. 2012, MNRAS, 420, 1977

Ramos-Larios, G., Guerrero, M. A., Vazquez, R., \& Phillips, J. P. 2013, arXiv: 1305.4211

Sahai, R., Morris, M. R., \& Villar, G. G. 2011, AJ, 141, 134

Schmidt-Voigt, M., \& Koppen, J. 1987, A\&A, 174, 223 
Schönberner, D., Jacob, R., Lehmann, H., et al. 2014, AN, 335, 378

Shimansky, V. V., Pozdnyakova, S. A., Borisov, N. V., et al. 2009, AstBu, 64, 349

Soker, N., \& Kastner, J. H. 2002, ApJ, 570, 245

Soker, N., \& Livio, M. 1994, ApJ, 421, 219

Soker, N., \& Rappaport, S. 2000, ApJ, 538, 241

Steffen, M., Schönberner, D., \& Warmuth, A. 2008, A\&A, 489, 173

Su, K. Y. L., Chu, Y.-H., Rieke, G. H., et al. 2007, ApJL, 657, L41
Toalá, J. A., \& Arthur, S. J. 2011, ApJ, 737, 100

Toalá, J. A., \& Arthur, S. J. 2014, MNRAS, 443, 3486

Toalá, J. A., Guerrero, M. A., Chu, Y.-H., et al. 2012, ApJ, 755, 77

Tocknell, J., De Marco, O., \& Wardle, M. 2014, MNRAS, 439, 2014

Vázquez, R., Miranda, L. F., Torrelles, J. M., et al. 2002, ApJ, 576, 860

Villaver, E., Manchado, A., \& García-Segura, G. 2002, ApJ, 581, 1204

Webster, B. L., Payne, P. W., Storey, J. W. V., \& Dopita, M. A. 1988, MNRAS, 235,533 